\title{
Random Access Based on Maximum Average Distance Code for Massive MTC in Cellular IoT Networks
}

\author{
Carlos A. Astudillo, Student Member, IEEE, Ekram Hossain, Fellow, IEEE, and Nelson L. S. da Fonseca, Senior \\ Member, IEEE
}

\begin{abstract}
Code-expanded Random Access (CeRA) is a promising technique for supporting massive machine-type communications in cellular IoT networks. However, its potentiality is limited by code ambiguity, which results from the inference of a larger number of codewords than those actually transmitted. In this letter, we propose a random access (RA) scheme to alleviate this problem by allowing devices to select the preambles to be transmitted considering a $q$-ary code with maximum average distance. Moreover, a CeRA decoding approach based on hypergraphs is proposed and an analytical model is derived. Numerical results show that the proposed scheme significantly increases the probability of successful channel access as well as resource utilization.
\end{abstract}

Index Terms-Cellular IoT networks, coding theory, Internet of things, massive machine-type communications, random access.

\section{INTRODUCTION}

$\mathbf{T}$ HE main challenge in supporting massive machine-type communications (mMTC) use case category in 5G networks is the handling of a massive number of devices in the random access (RA) procedure. 5G networks rely on New Radio (NR) and cellular IoT (CIoT) network technologies, such as LTE-M and NB-IoT, for supporting mMTC. These technologies employ RA procedures based on grants, which require the transmission of an orthogonal preamble sequence before the reception of a grant for data transmission. One of the limiting factors is the reduced number of RA contention resources (e.g. up to 48 orthogonal preambles in NB-IoT or 64 in LTE-M/NR), which can lead to a large number of transmission collisions. Such collisions decreases the probability of successful channel access and reduces the efficiency in the usage of available resources.

Several improvements to grant-based RA procedures have been proposed, including overload control, non-orthogonal multiple access (NOMA) transmissions, and collision detection and resolution. Some proposals adopt the transmission of more than one preamble [1]-[3] in order to overcome the limitation of RA resources as well as reduce the chances that two or more devices select the same RA contention resource. One alternative is to aggregate two preambles with different Zadoff-Chu (ZC) root sequences in the same RA subframe [1], which may require hardware modification and can considerably increase the multiple-access interference due to the loss of orthogonality. Moreover, this cannot be applied to the NB-IoT preamble, which is based on a single-tone waveform combined with frequency hopping.

This work was supported by the São Paulo Research Foundation (FAPESP) under grant number 15/24494-8 and 19/22065-3, and the Government of Canada through the Emerging Leaders in the Americas Program (ELAP).

C. A. Astudillo and N. L. S da Fonseca are with the Institute of Computing, University of Campinas 13083-852, Brazil. E. Hossain is with the Department of Electrical and Computer Engineering, University of Manitoba, Winnipeg, MB R3T 5V6, Canada (e-mails: castudillo@lrc.ic.unicamp.br, ekram.hossain@umanitoba.ca, nfonseca@ic.unicamp.br).

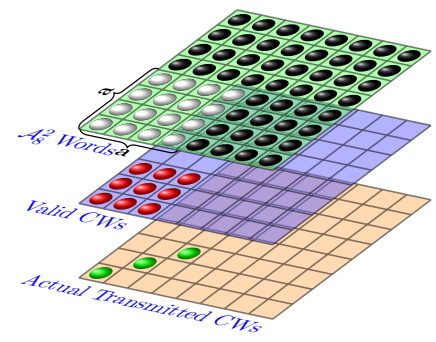

(a) CeRA scheme

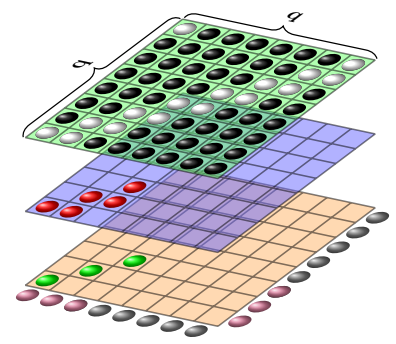

(b) OptCeRA scheme
Fig. 1: Illustration of CeRA decoding and the code ambiguity problem. 2 RA subframes per superframe, 8 preambles available per subframe, 16 codewords (white balls), and 3 transmitted codewords (green balls); black balls denote words in $\mathcal{A}_{8}^{2}$ not in the code; gray and red balls represent, respectively, the idle and detected preambles; and blue balls denote the valid codewords.

A more attractive alternative is code-expanded RA (CeRA) [2], [3], in which each device performs random access by using consecutive transmissions of randomly selected preambles over $n$ RA subframes, to form an RA superframe. At every RA subframe, a preamble is randomly selected from the set of $a$ allowed preambles. This sequence of preambles over the superframe is interpreted as a codeword of length $n$. Uplink resources for data transmission are allocated on the basis of the codewords inferred by the base station (BS) in the superframe.

However, in this scheme, individual codewords are not received by the $\mathrm{BS}$, where the preamble transmission from several devices at each subframe is identified. Consequently, a larger number of codewords can be considered valid than those actually transmitted in a superframe. For example, consider a CeRA scheme with two RA subframe per superframe, four preambles allowed in a subframe among the eight available preambles, and 16 codewords as illustrated in Fig. 1a. The possible codewords for the devices to perform RA are represented by white balls. Consider that 3 different codewords (green balls) are transmitted by the devices in a superframe. The BS detects the preambles transmitted at each subframe of a superframe (red balls) and generates the set of inferred valid codewords (blue balls). Note that all possible combinations of preambles received in different subframes within the superframe are regarded as valid. This problem is known as the code ambiguity problem [3] and it impacts greatly on the utilization of uplink resources as well as on success probability under resource constraints.

Only recently, various solutions have been proposed for the code ambiguity problem [2]-[5]. It has been proposed that the medium access control (MAC) layer adapts the number of preambles allowed in a subframe considering the device load in order to reduce the number of possible combinations of preambles that can be inferred [2], [3]. However, this strategy does not directly tackle the problem. On the other hand, solutions using massive multiple-input multiple output 
TABLE I: Notation

\begin{tabular}{l||l}
\hline Symbol & Definition \\
\hline $\mathcal{I}$ & Index set of the codeword coordinates \\
$\mathcal{T}$ & Index set of the code symbols \\
$\mathcal{T}$ & Index set of the codewords \\
$P_{\text {alloc }}$ & Resource allocation probability \\
$P_{N}$ & Codeword non-colission probability \\
$P_{S}$ & RA success probability \\
$\eta$ & Grant utilization \\
$K$ & Number of contending devices \\
$M$ & Number of available codewords \\
$N$ & Number of words in $\mathcal{A} n$ \\
$R$ & Number of available resources per superframe \\
$V$ & Number of inferred valid codewords \\
$a$ & Number of allowed preambles \\
$k$ & Multiplicative factor of available preambles \\
$n$ & Number of RA subframes per superframe \\
$q$ & Number of available preambles \\
$r$ & Number of preambles used in the code \\
\hline
\end{tabular}

(mMIMO) [4], [5] assume perfect channel estimation and spatial orthogonality which are hard to achieve in practice, especially in mMTC scenarios. These solutions increase the system complexity and cannot be used by CIoT technologies since they employ low resolution MIMO settings and either frequency division duplexing or half-duplex. The code ambiguity problem must be addressed to exploit the true potentiality of CeRA and enable its wide adoption for mMTC in 5G and beyond networks.

This paper proposes OptCeRA, a CeRA scheme based on MAC coding to alleviate the code ambiguity problem. In the proposed scheme, the transmitted preambles are chosen on the basis of a new $q$-ary maximum average distance (MAD) code, which minimizes the similarity between codewords. Moreover, this paper introduces an encoding procedure for a MAD code, and a decoding approach for CeRA schemes based on hypergraphs, as well as an analytical model for CeRA schemes. Table [ shows the notation used in the paper.

\section{Maximum Average Distance Codes and their Application to Code-Expanded RA}

This section introduces the OptCeRA scheme to tackle the code ambiguity problem. OptCeRA optimizes the code used to perform consecutive preamble transmissions. For some fixed value of $n$, when the number of available codewords is reduced, redundancy is introduced, imposing constraints to the transmitted messages (codewords) in a way that just a fraction of all possible received messages (all combinations of the received preambles at each subframe) are valid codewords. By reducing the similarity between the codewords in the code, the number of inferred valid codewords can be reduced as well. This reduction can be formulated as the maximization of the average Hamming distance between the codewords in a code.

In contrast to the existing CeRA schemes, in which the codeword is formed by a sequence of preambles selected randomly from the $a$ allowed preambles, in the OptCeRA scheme, the codeword is first randomly selected from a code $C$ with maximum average Hamming distance (a MAD code), considering all the $q$ available preambles, i.e. $C \subseteq \mathcal{A}_{q}^{n}$, where $\mathcal{A}_{q}$ is a finite set of $q$ symbols (a $q$-ary alphabet). Then, a preamble is transmitted at the $i$ th subframe according to the $i$ th coordinate of the chosen codeword. Using the OptCeRA approach, the CeRA scheme in [3] becomes equivalent to randomly selecting a codeword from a code $C=\mathcal{A}_{a}^{n}$.

Since a $q$-ary code of a given size and length with the MAD property is unknown, we formulate the MAD code problem, characterize these codes, and show their construction. Moreover, we present a hypergraph representation of codes for CeRA which facilitates their storage, analysis, and processing. Finally, we introduce a simple method based on the proposed hypergraph representation to perform decoding in CeRA.

\section{A. Preliminaries}

Let $n$ and $q$ be positive integers and let $\mathcal{A}_{q}=\{0,1, \ldots, q-1\}$ be a finite set, where $q \geq 2$ denotes $\left|\mathcal{A}_{q}\right|$. A code $C$ of block-length $n$ over a $q$-ary alphabet $\mathcal{A}_{q}$ is a subset of $\mathcal{A}_{q}^{n}$. The elements of $\mathcal{A}_{q}^{n}$, also called words, are all the ordered $n$-tuples over $\mathcal{A}_{q}$ of the form $\mathbf{x}=x_{n} x_{n-1} \cdots x_{1}$, where $x_{i} \in \mathcal{A}_{q}$; the elements of $C$ are called codewords; and $M=|C|$ denotes the size of $C$.

The Hamming distance between two $n$-tuples $\mathbf{x}, \mathbf{y} \in \mathcal{A}_{q}^{n}$ is defined as the number of coordinates in which $\mathbf{x}$ and $\mathbf{y}$ differ and it is given by $H(\mathbf{x}, \mathbf{y})=\mid\left\{i: i \in\{1,2, \cdots, n\}, x_{i} \neq\right.$ $\left.y_{i}\right\} \mid$. The average Hamming distance of $C$ is calculated as $d_{H}(C)=1 / M^{2} \cdot \sum_{\mathbf{x} \in C} \sum_{\mathbf{y} \in C} H(\mathbf{x}, \mathbf{y})[6]$.

\section{B. Problem Formulation}

The MAD code problem can be stated as follows: given $\mathcal{A}_{q}^{n}$ of size $N=\left|\mathcal{A}_{q}^{n}\right|$, select a code $C \subseteq \mathcal{A}_{q}^{n}$ of size $M$, where $M \leq N$, with maximum average distance among all the possible codes of size $M$ in $\mathcal{A}_{q}^{n}$. Thus, a discrete optimization problem is formally defined as:

$$
\begin{array}{cc}
\mathcal{P} 1 \quad \underset{C \subseteq A_{q}^{n}}{\operatorname{maximize}} & d_{H}(C) \\
& \\
\text { subject to } & |C|=M .
\end{array}
$$

An optimal solution for this problem is denominated an $(n, M)_{q}$-MAD code. Note that $\mathcal{P} 1$ may have more than one possible optimal solution. Since all the devices in OptCeRA must use the same code, a systematic solution must be implemented by the devices and the BS. The problem $\mathcal{P} 1$ is the wellknown optimal subset selection problem, which is an NP-hard problem. Exhaustive search for solving it, however, would be prohibitive, even for moderate instances of the posed problem. Even though there exist numerical methods that can be applied to obtain a solution for $\mathcal{P}_{1}$ in polynomial time, theoretical results are exploited here for our objective function to change the domain of the problem from the code itself to a general property of the code, namely, the distribution of the alphabet elements (code symbols) on each codeword coordinate in $C$, denoted by $\pi_{i}=\left\{\pi_{i}(j): j \in \mathcal{A}_{q}\right\}, i \in\{1,2, \cdots, n\}$. In this way, we are able to obtain an optimal distribution that characterizes the MAD codes. Such characterization makes it possible for us to present a systematic solution, thus facilitating the practical design of the scheme.

From [6], we have $d_{H}(C)$ equals

$$
\mathbb{E}\left[H\left(X_{C}, Y_{C}\right)\right]=\sum_{i=1}^{n}\left(1-\sum_{j=0}^{q-1} \pi_{i}(j)^{2}\right),
$$


where $X_{C}$ and $Y_{C}$ are two i.i.d. random $n$-tuples with common distribution $P_{C}=\left\{P_{C}(\mathbf{x}): \mathbf{x} \in \mathcal{A}_{q}^{n}\right\}, P_{C}(\mathbf{x})$ equals $1 / M, \forall \mathbf{x} \in C$ and 0 otherwise; $\pi_{i}(j)=\underset{\substack{\mathbf{x} \in \mathcal{A}_{q}^{n} \\ x_{i}=j}}{ } P_{C}(\mathbf{x})$, and

$$
\sum_{j=0}^{q-1} \pi_{i}(j)=1, \forall i=1,2, \cdots, n .
$$

By using (2) and (3), we formulate the following optimization problem:

$$
\begin{gathered}
\mathcal{P} 2 \quad \underset{\left\{\pi_{i}(j)\right\}}{\operatorname{maximize}} \mathbb{E}\left[H\left(X_{C}, Y_{C}\right)\right] \\
\text { subject to }
\end{gathered}
$$

We present next an optimal solution for $\mathcal{P} 1$ by constructing a code with the optimal distribution obtained from $\mathcal{P} 2$.

\section{The OptCeRA code: A MAD Code Construction}

Since the granularity of the RA configuration parameters is limited due to signaling overhead 1 , we restrict ourselves to code sizes of multiple of $q$. By assuming this restriction, Theorem 1 gives the optimal solution for $\mathcal{P} 2$.

Theorem 1 (Characterization of MAD codes). A code $C \subseteq \mathcal{A}_{q}^{n}$ of size $M$ multiple of $q$ is an $(n, M)_{q}$-MAD code if and only if $\pi_{i}(j)$ equals $1 / q$ for all $i=1,2, \cdots, n$ and $j=0,1, \cdots, q-1$.

Proof. We prove this theorem by using Fu et al.'s theoretical results for the average Hamming distance of a code [6]. The objective function in (4) is upper bounded by $n(1-1 / q)$ [6. Theorem 1], and equality with this upper bound holds if and only if $\pi_{i}(j)=1 / q, \forall i, j[6$, Theorem 4$] 2$. Given that $\pi_{i}$ is the distribution of the alphabet elements on coordinate $i$ over all codewords in the code, $\pi_{i}(j)=1 / q, \forall i, j$ implies that the code size is a multiple of $q$ and Theorem 1 follows.

Theorem 11 gives the distribution $\pi_{i}$ of a MAD code. However, a code with that property must be constructed to obtain a solution for $\mathcal{P} 1$. A specific construction of an $(n, M)_{q^{-}}$ MAD code is thus provided here, the $(n, q, k)$-OptCeRA code, where $k=M / q, k \in\left\{1,2, \cdots, q^{n-1}\right\}$, is a multiplicative factor of the available preambles of the OptCeRA scheme.

The rationale of the proposed method follows that in [7]. Let $\mathcal{T}$ be the index set of the codewords in code $C \subseteq \mathcal{A}_{q}^{n}$. $\mathcal{T}=\{0,1, \cdots, M-1\}, M \leq N=q^{n}$. An integer $t \in \mathcal{T}$ has a unique representation $t=a_{n} q^{n-1}+a_{n-1} q^{n-2}+\cdots+a_{1} q^{0}$, where $a_{i} \in \mathbb{Z}_{q}$. Thus, there is a unique correspondence between each $t \in \mathcal{T}$ and an $n$-tuple of $\mathbb{Z}_{q}^{n} \mathbf{a}=\left(a_{n}, a_{n-1}, \cdots, a_{1}\right)$. We can always arrange the elements of $\mathbb{Z}_{q}^{n}$ in the natural order of the number they represent. Based on this $q$-ary representation of integers $0,1, \cdots, M-1$, if $\mathbf{c}=\left(c_{n}, c_{n-1}, \cdots, c_{1}\right)$ is the $t$ th code vector of an $(n, q, k)$-OptCeRA code, then $\mathbf{c}$ is obtained from the corresponding $n$-tuple $\mathbf{a}=\left(a_{n}, a_{n-1}, \cdots, a_{1}\right)$ by employing the following mapping:

$$
c_{i}=\sum_{l=1}^{i} a_{l} \quad(\bmod q), \forall i=1,2, \cdots, n .
$$

As an illustration, Table [II gives the above transformation for the $(2,8, k)$-OptCeRA code, $k=1,2$. Since $\pi_{i}(j)=1 / 8$,

${ }^{1}$ For instance, even though there are up to 64 preambles available for RA in NR or LTE-M, just 14 possible values can be configured.

${ }^{2}$ Even though the theorem just claims the necessary condition, its proof shows that it is also sufficient.
TABLE II: Transformation for the $(2,8, k)$-OptCeRA code

\begin{tabular}{cccc|cccc}
\hline $\mathbf{k}$ & $\mathbf{C W}$ & $\boldsymbol{Z}_{\mathbf{8}}^{\mathbf{2}}$ & $\mathbf{O p t C e R A}$ & $\mathbf{k}$ & $\mathbf{C W}$ & $\boldsymbol{Z}_{\mathbf{8}}^{\mathbf{2}}$ & OptCeRA \\
\hline \hline \multirow{6}{*}{1 or 2} & 0 & 00 & 00 & & 8 & 10 & 10 \\
& 1 & 01 & 11 & & 9 & 11 & 21 \\
& 2 & 02 & 22 & & 10 & 12 & 32 \\
& 3 & 03 & 33 & 2 & 11 & 13 & 43 \\
& 4 & 04 & 44 & & 12 & 14 & 54 \\
& 5 & 05 & 55 & & 13 & 15 & 65 \\
& 6 & 06 & 66 & & 14 & 16 & 76 \\
& 7 & 07 & 77 & & 15 & 17 & 07 \\
\hline
\end{tabular}

$\forall i, j$, the resulting code is a $(2,8 \cdot k)_{8}$-MAD code, assured by Theorem 1. Note that a simple encoding procedure for the OptCeRA scheme can be derived from this construction. In the beginning of a superframe, a device randomly selects an integer between 0 and $M-1$, calculates its $q$-ary representation, and applies the transformation in (5) to obtain a random OptCeRA codeword, that defines which preamble is transmitted at each RA subframe, as explained in the beginning of this section.

\section{Hypergraph Representation of Codes for CeRA Schemes}

Let $r \leq q$ be the number of preambles used in the code 3 Let $\mathcal{I}=\{1,2, \cdots, n\}$ and $\mathcal{T}=\{0,1, \cdots, r-1\}$, be the index set of the codeword coordinates and code symbols, respectively. We model a code $C \subseteq \mathcal{A}_{r}^{n}$ of size $M$ for CeRA as a simple $n$-partite $n$-uniform hypergraph $H=(\mathcal{X}, \mathcal{E})$, where $\mathcal{X}=\left\{x_{i, j}: i \in \mathcal{I} \wedge j \in \mathcal{J}\right\}$ is a finite set of vertices, and $\mathcal{E}=\left\{E_{t}: t \in \mathcal{T}\right\}$ is a family of non-empty subsets of $\mathcal{X}$ called hyperedges. $X$ can be partitioned into $n$ disjoint subsets $\mathcal{X}_{1}, \mathcal{X}_{2}, \cdots, \mathcal{X}_{n}$ (n-partite), and each hyperedge $E_{t} \in \mathcal{E}$ contains $n$ vertices ( $n$-uniform), exactly one vertex from each subset of $\mathcal{X}$, i.e. $E_{t}=\left\{e_{1}, e_{2}, \cdots, e_{n}\right\}$, where $e_{i} \in \mathcal{X}_{i}, \forall i$. In this model, $\mathcal{X}_{i}=\left\{x_{i, j}: j \in \mathcal{J}\right\}$ represents the set of preambles used at $i$ th RA subframe, and $\mathcal{E}$ represents the codewords of $C$. Thus, there is a hyperedge $E=\left\{e_{1}, e_{2}, \cdots, e_{n}\right\} \in \mathcal{E}$ if and only if there is a codeword $\mathbf{c}=c_{n} c_{n-1} \cdots c_{1} \in C$ such that $e_{i}=x_{i, c_{i}}, \forall i$.

\section{E. Code-expanded RA Decoding}

Unlike traditional decoding schemes in which the input of the decoding process is a received message and the output is the transmitted codeword or a list of possible candidates, the decoding process in CeRA has as input the set of received code symbols (detected preambles) at each codeword coordinate (RA subframe) and as output the list of inferred valid codewords 4 , which calls for innovative decoding strategies. In this paper, we formulate the code-expanded RA decoding as operations over the hypergraph representation of the code, as given in Lemma 1 . Note that Lemma 1 applies to any existing code for code-expanded RA.

Definition 1 (Set of inferred valid codewords). Given $y=$ $\left\{\mathcal{Y}_{i}: i \in \mathcal{I}\right\} \subseteq \mathcal{X}$, where $\mathcal{Y}_{i}$ is the set of preambles detected at $i$ th RA subframe, the set of valid codewords inferred by the BS in a superframe is given by all codewords of the form $\mathbf{c}=c_{n} c_{n-1} \cdots c_{1} \in C$ such that $x_{i, c_{i}} \in \mathcal{Y}_{i}, \forall i$.

Definition 2 (Induced subhypergraph). An induced subhypergraph of a hypergraph $H=(\mathcal{X}, \mathcal{E})$, is the hypergraph $H_{y}=$ $\left(\mathcal{Y}, \mathcal{E}^{\prime}\right)$, where $\mathcal{Y} \subseteq \mathcal{X}$ and a hyperedge $E \in \mathcal{E}$ is in $\mathcal{E}^{\prime}$ if and only if all the vertices of $E$ are in $\mathcal{Y}$.

${ }^{3} r$ equals $a=M^{1 / n}$ for Multipreamble RA [3] and $q$ for OptCeRA.

${ }^{4} \mathrm{CeRA}$ can be seen as unsourced RA $|8|$ at the MAC layer, in which the objective is to communicate a message to get channel access. 
Lemma 1 (Decoding in CeRA). Given a code $C \subseteq \mathcal{A}_{r}^{n}$ with $H=(\mathcal{X}, \mathcal{E})$ and $\mathcal{Y}=\left\{\mathcal{Y}_{i}: i \in \mathcal{I}\right\} \subseteq \mathcal{X}$, where $\mathcal{Y}_{i}$ is the set of preambles detected at $i$ th RA subframe of a given superframe, the set of valid codewords inferred in the superframe is given by the hyperedge set of the subhypergraph induced by $\mathcal{y}$.

Proof. Assume $H=(X, \mathcal{E})$ of a code $C \subseteq \mathcal{A}_{r}^{n}$. Let $\mathcal{Y}=\left\{\mathcal{Y}_{i}: i \in\right.$ $\mathcal{I}\} \subseteq \mathcal{X}$ be the set of preambles detected at each RA subframe of a superframe. From Definition 2 and the model described in Section II-D there is a hyperedge $E=\left\{e_{1}, e_{2}, \cdots, e_{n}\right\} \in \mathcal{E}$ in $H_{y}$ if and only if $e_{i} \in \mathcal{Y}_{i}, \forall i$. Let us call such a hyperedge a covered hyperedge and the represented codeword $\mathbf{c}=c_{n} c_{n-1} \cdots c_{1} \in \mathcal{C}$ such that $e_{i}=x_{i, c_{i}}, \forall i$, a covered codeword. Thus, using Definition 1 and the above argument, the set of inferred valid codewords is equal to the set of covered codewords, and Lemma 1 follows.

\section{F. Example}

The following example illustrates the advantage of using OptCeRA (Fig. 1b) when compared to CeRA (Fig. [1a) [2], [3]. It can be noted that although the two schemes have the same number of available codewords (white balls) and transmitted codewords (green balls), the number of codewords inferred (blue balls) by OptCeRA is less than that inferred by CeRA, which evinces the greater efficiency in resource utilization achieved by the proposed scheme.

\section{Analytical Model for CeRA Schemes}

We introduce an analytical model for CeRA schemes which covers both the Multipreamble RA scheme in [3] and the proposed OptCeRA scheme. Given that the impact of the code ambiguity problem on resource utilization efficiency has been neglected in the literature, we include not only the RA success probability, but also grant utilization in the model.

Assume that $K$ devices employ a CeRA scheme with $n$ RA subframes per superframe. Let $X$ denote the random variable (RV) of the number of devices contending per codeword in a given superframe. The probability distribution of $X$ follows a binomial distribution with parameters $K$ and $p=1 / M$, where $M$ is the number of available codewords [2]. Thus, $\mathbb{P}(X=m)=$ $\mathbb{B}_{m}(K, p)=\left(\begin{array}{l}K \\ m\end{array}\right) p^{m}(1-p)^{K-m}$. Note that $M$ equals $q \cdot k$ for the OptCeRA scheme and $a^{n}$ for the Multipreamble RA scheme, where $a \in\{1,2, \cdots, q\}$. The expected number of codewords chosen by at least one device (selected) and by a single device (non-collided) are given by $N_{C}=M \cdot\left[1-\mathbb{B}_{0}(K, p)\right]=M \cdot[1-$ $\left.(1-p)^{K}\right]$ and $N_{S}=M \cdot \mathbb{B}_{1}(K, p)=K(1-p)^{K-1}$, respectively.

The codeword non-colission probability for CeRA schemes is $P_{N}=N_{S} / K=(1-p)^{K-1}$, and the RA success probability can be calculated as $P_{S}=P_{N} \cdot P_{\text {alloc }}$ [3] where $P_{\text {alloc }}$ is the resource allocation probability. Let $V$ be the RV of the number of inferred valid codewords. Since $V$ can be greater than the number of available resources in a superframe $(R)$, not all the inferred valid codewords can be served in a given superframe. Thus, $P_{\text {alloc }}$ equals $R / \mathbb{E}[V]$ if $\mathbb{E}[V]>R$, or equals 1 otherwise [3]. Theorem 2 gives $\mathbb{E}[V]$ for the case when $n=2$, which is of main interest due to the practical reasons in Section IV The proof of Theorem 2 is described in a way that, given the code, $\mathbb{E}[V]$ can be calculated numerically for a general $n$ value.

${ }^{5}$ We consider a device to be successful if its codeword is served and noncollided. Since $K$ is the number of contending devices perceived by the BS (see Section IV], the codeword detection probability is not included.
Theorem 2 (Expectation of $V$ ). If $K$ devices are trying RA simultaneously employing a code-expanded RA scheme with code $C \subseteq \mathcal{A}_{r}^{2}$ of size $M, r \leq q$, and $\pi_{i}(j)$ equals $1 / r$, for all $i=1,2$ and $j=0,1, \cdots, r-1$, then $\mathbb{E}[V]$ is calculated as

$$
\mathbb{E}[V]=M \cdot\left[1-\left[2(1-1 / r)^{K}-(1-2 / r+1 / M)^{K}\right]\right] .
$$

Proof. Consider a network employing a code-expanded RA scheme. Suppose each of $K$ devices trying RA in a given superframe randomly selects a codeword from a code $C \subseteq \mathcal{A}_{r}^{n}$ of size $M$. Let $H=(X, \mathcal{E})$ be the hypergraph representation of $C$. In the superframe, the base station detects a set of preambles $\boldsymbol{Y}=\left\{\mathcal{Y}_{i}: i \in \mathcal{I}\right\}$. By Lemma 1, the set of inferred valid codewords can be obtained from the set of hyperedges of $H_{y}$. Let us call an hyperedge covered if it is in $H_{y}$ and noncovered otherwise. Let $A_{t}$ be the event that $E_{t} \in \mathcal{E}$ is covered and $A_{t}^{c}$ its complement; let $I_{A_{t}^{c}}$ be the indicator function of $A_{t}^{c}$. Let $V=\sum_{t \in \mathcal{T}} 1-I_{A_{t}^{c}}$ be the RV of the number of covered hyperedges or, equivalently, the number of inferred valid codewords, and $\mathbb{E}[V]=\sum_{t \in \mathcal{T}} 1-\mathbb{P}\left(A_{t}^{c}\right)$ the expectation of $V$, where $\mathbb{P}\left(A_{t}^{c}\right)$ is the probability of $A_{t}^{c}$. Let $A_{t, i}^{c}$ be the event that $e_{i} \in E_{t}$ is not in $\mathcal{Y}_{i}$, i.e. the $i$ th coordinate of the $t$ th codeword is not in the set of preambles detected at $i$ th RA subframe. Since an element $E_{t} \in \mathcal{E}$ is in $H_{y}$ if and only if all the vertices of $E_{t}$ are in $\mathcal{Y}, A_{t}=\cap_{i=1}^{n} A_{t, i}$ and $A_{t}^{c}=\cup_{i=1}^{n} A_{t, i}^{c}$. By applying the inclusion-exclusion principle, $\mathbb{P}\left(A_{t}^{c}=\bigcup_{i=1}^{n} A_{t, i}^{c}\right)=\sum_{\emptyset \neq \mathcal{S} \subseteq\{1,2, \cdots, n\}}(-1)^{|\mathcal{S}|-1} \mathbb{P}\left(\cap_{i \in \mathcal{S}} A_{t, i}^{c}\right)$. For the case $n=2, \mathbb{P}\left(A_{t}^{c}\right)=\mathbb{P}\left(A_{t, 1}^{c}\right)+\mathbb{P}\left(A_{t, 2}^{c}\right)-\mathbb{P}\left(A_{t, 1}^{c} \cap A_{t, 2}^{c}\right)$. Moreover, if $\pi_{i}(j)=1 / r, \forall i, j$, then, we have $M=r \cdot k$, and, $\forall t, i, \mathbb{P}\left(A_{t, i}^{c}\right)=(1-k / M)^{K}$ and $\mathbb{P}\left(A_{t, 1}^{c} \cap A_{t, 2}^{c}\right)=(1-(2 k-1) / M)^{K}$. Note that $k$ and $2 k-1$ are the number of codewords in $C$ that satisfy the complement of the event for which probability has been calculated by considering a single device. Theorem 2 follows after inserting $\mathbb{P}\left(A_{t}^{c}\right)$ into $\mathbb{E}[V]$, summing up over all $t \in \mathcal{T}$, and substituting $k$ by $M / r$.

Finally, let $\eta$ be the grant utilization defined as $\eta=U / G$, where $U$ is the number of grants actually used and $G$ is the number of uplink grants issued for RA devices in a superframe; $\mathbb{E}[\eta]=\mathbb{E}[U / G]=\mathbb{E}\left[C \cdot P_{\text {alloc }} / V \cdot P_{\text {alloc }}\right] \approx \mathbb{E}[C] / \mathbb{E}[V]$, where $C$ is the number of grants actually needed. Thus, $\mathbb{E}[C]$ equals $N_{C}$ for both the Multipreamble RA and OptCeRA schemes.

\section{Practical Considerations}

The OptCeRA scheme can be applied to existing technologies, e.g. 5G NR, NB-IoT, LTE-M, LTE-A, and LTE, in a straightforward way. All these technologies use system information broadcast (SIB) messages to dynamically configure the RA channel (RACH) at some subframes (RA subframes) by setting the value of the PRACH configuration index parameter as well as allocating $q$ preambles for contention-based RA.

The values of $n, q$, and $k$ need to be set to configure the OptCeRA scheme, but caution needs to be taken since both energy consumption and access delay may increase with the value of $n$. When $n=2$, up to $64^{2}$ codewords can be provided which is greater than the available control/data resources in the aforementioned technologies. Thus, the value of $n$ can be fixed to 2. Moreover, this also helps to reduce the signaling overhead. While the value of $n$ can be preconfigured, the value of $k$ needs to be added to an SIB message. 


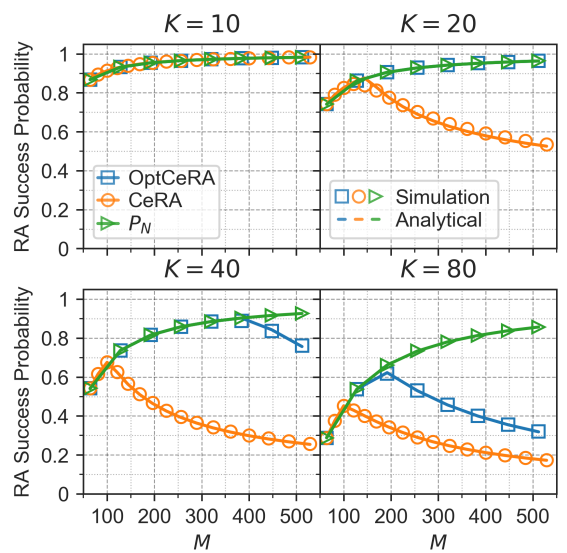

(a) RA success probability $\left(P_{S}\right)$
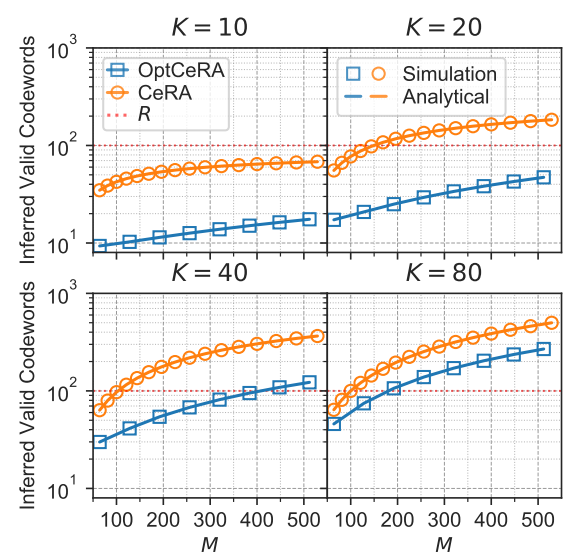

(b) Number of inferred valid codewords $(\mathbb{E}[V])$
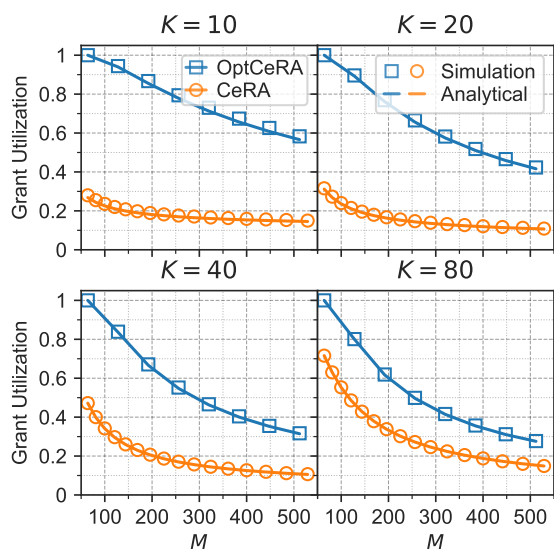

(c) Grant utilization $(\mathbb{E}[\eta])$

Fig. 2: Performance evaluation results

For using the analytical model in Section III] the value of $K$ is needed, which can be estimated at the BS by using existing approaches based on idle and detected preambles. In this way, $K$ can be regarded as the number of devices contending with a codeword whose preambles are detected in a superframe.

\section{Numerical Results}

This section compares the Multipreamble RA [3] (hereinafter referred to as CeRA) and OptCeRA schemes. The proposed analytical model is also validated by comparing the analytical and simulation results. Results are presented as a function of $M^{6}$ and $K$ for $q=64, n=2$, and $R=1007$ The proposed OptCeRA scheme outperforms the CeRA scheme for all device loads and code sizes considering $P_{S}$ (Fig. 2a), $\mathbb{E}[V]$ (Fig. 2b) and $\mathbb{E}[\eta]$ (Fig. 2c), and achieves more than 50\%, $100 \%$ and $300 \%$ performance gain, respectively.

When $P_{\text {alloc }}^{C e R A}=1(\mathbb{E}[V] \leq R)$, the two schemes produce the same $P_{S}$ values (Fig. 2a), but the granted resources are utilized very inefficiently when the CeRA scheme is employed (Fig. 2c). Moreover, a cellular network typically has two groups of devices: random access-based devices and scheduling-based devices (e.g. based on semi-persistent or dynamic scheduling). Hence, the low grant utilization of the CeRA scheme may significantly affect the performance of both groups of devices in a coexistence scenario.

Furthermore, when $P_{\text {alloc }}<1(\mathbb{E}[V]>R)$ in each scheme, its $P_{S}$ value decreases as $M$ increases even though $P_{N}$ increases. This situation occurs because the number of inferred valid codewords is larger than the available resources (Fig. $2 \mathrm{~b}$ ), forcing the network to randomly allocate the available resources among the inferred valid codewords. Thus, some allocated resources are not actually used by any device while a certain portion of the devices waiting for an uplink grant does not receive response, even though their preamble transmission are correctly received at each RA subframe. This may strongly impact on the performance of code-expanded RA in a limited

${ }^{6} M$ equals $a^{n}$ and $q \cdot k$ for the CeRA and OptCeRA schemes, respectively. To assign values to $M$ in Fig. 2 we used $a \in\{8,9, \cdots, 23\}$ and $k \in$ $\{1,2, \cdots, 8\}$.

${ }^{7}$ Considering an LTE-M network with a 2-subframe RA superframe every 10 subframes, and 6 uplink resources available per subframe not being a RA subframe, $R=(10-2) \cdot 6=48$. Moreover, considering a NOMA technique with $100 \%$-overloading [9], we obtain roughly 100 uplink resources per RA superframe, which is a realistic value for $R$ in a CIoT network. Higher values of $R$, however, are possible by decreasing the frequency of the RA subframes. resource regime. However, for a given number of devices, this happens with a higher value of $M$ when OptCeRA scheme is used, allowing OptCeRA scheme to support higher $P_{S}$ values than does the CeRA scheme.

All of these gains are achieved thanks to the proposed optimized OptCeRA code. It significantly reduces the number of codewords that the network infers, effectively alleviating the code ambiguity problem. Moreover, these results show that the OptCeRA scheme, as well as CeRA and other RA schemes ([2], [3]), have an optimal point of operation which defines the system parameter values (e.g. $M$ ) and depends on the device load $(K)$. Such an optimal point can be derived by using the analytical model proposed here, which is in full agreement with Monte Carlo simulation results ( $10^{5}$ iterations).

\section{Conclusion}

This letter has addressed the code ambiguity problem in code-expanded RA for mMTC by allowing devices to select codewords from a novel code with maximum average distance. The proposed scheme reduces the number of valid codewords that can be inferred, and greatly increases the RA success probability as well as the efficiency in resource utilization. The proposed maximum average distance code is likely to find other innovative applications.

\section{REFERENCES}

[1] A. E. Mostafa et al., "Aggregate preamble sequence design for massive machine-type communications in 5G networks," in 2018 IEEE Global Commun. Conf. (GLOBECOM), 2018, pp. 1-6.

[2] H. Thomsen et al., "Code-expanded radio access protocol for machineto-machine communications," Trans. on Emerging Telecommun. Techn., vol. 24 , no. 4 , pp. $355-365,2013$.

[3] S. Vural et al., "Success probability of multiple-preamble-based singleattempt random access to mobile networks," IEEE Commun. Letters, vol. 21, no. 8, pp. 1755-1758, Aug 2017.

[4] H. Jiang, J. Ding, and D. Qu, "Improved success probability of codeexpanded single-attempt random access by massive MIMO," in 201928 th Wireless and Optical Commun. Conf. (WOCC), May 2019, pp. 1-5.

[5] H. Jiang et al., "Multiple preambles for high success rate of grant-free random access with massive MIMO," IEEE Trans. on Wireless Commun., vol. 18, no. 10, pp. 4779-4789, Oct 2019.

[6] Fang-Wei Fu, T. Klove, and Shi-Yi Shen, "On the Hamming distance between two i.i.d. random n-tuples over a finite set," IEEE Transactions on Information Theory, vol. 45, no. 2, pp. 803-807, March 1999.

[7] B. D. Sharma and R. K. Khann, "On m-ary gray codes," Information Sciences, vol. 15, no. 1, pp. 31 - 43, 1978.

[8] Y. Polyanskiy, "A perspective on massive random-access," in 2017 IEEE Int. Symp. Inf. Theory (ISIT), 2017, pp. 2523-2527.

[9] Y. Liang et al., "Non-orthogonal random access for 5G networks," IEEE Trans. on Wireless Commun., vol. 16, no. 7, pp. 4817-4831, 2017. 